\title{
Effects of Nasal Continuous Positive Airway Pressure on the Glomerular Filtration Rate in Patients with Obstructive Sleep Apnea Syndrome
}

\author{
Seiji Koga, Satoshi Ikeda, Tomohiko Yasunaga, Tomoo Nakata and Koji Maemura
}

\begin{abstract}
Objective Previous studies have shown a possible role for obstructive sleep apnea syndrome (OSAS) in the development and/or progression of chronic kidney disease (CKD). However, the impact of treatment for OSAS on CKD has not been clarified. The aim of this study was to investigate the influence of OSAS and the short-term effects of nasal continuous positive airway pressure (CPAP) on the estimated glomerular filtration rate (eGFR).

Methods We evaluated 38 men with OSAS diagnosed on polysomnography. The associations between the eGFR and patient characteristics, including polysomnographic parameters, were evaluated. A multivariate linear regression analysis was performed to determine the independent variables associated with eGFR. We reassessed the polysomnographic data and eGFR values after three months of CPAP treatment.

Results The mean serum creatinine level was $0.83 \pm 0.10 \mathrm{mg} / \mathrm{dL}$ and the mean eGFR was $77.3 \pm 12.0 \mathrm{~mL} /$ $\min / 1.73 \mathrm{~m}^{2}$. A univariate analysis revealed that an older age $(\mathrm{p}<0.001)$, a longer mean apnea duration $(\mathrm{p}=$ $0.006)$ and BMI $(\mathrm{p}=0.022)$ were significantly associated with lower eGFRs. A multivariate linear regression analysis showed that the independent factors associated with lower eGFRs were older age $(\mathrm{p}<0.001)$ and a longer mean apnea duration $(\mathrm{p}=0.048)$. Three months after CPAP treatment, there were significant decreases in the serum creatinine levels $(\mathrm{p}=0.013)$ and increases in eGFR $(\mathrm{p}=0.014)$.

Conclusion OSAS, especially that associated with an older age and a longer mean apnea duration, may contribute to lowering eGFR values, which can be reversed by CPAP treatment.
\end{abstract}

Key words: chronic kidney disease, continuous positive airway pressure, glomerular filtration rate, obstructive sleep apnea

(Intern Med 52: 345-349, 2013)

(DOI: 10.2169/internalmedicine.52.8468)

\section{Introduction}

Obstructive sleep apnea syndrome (OSAS) is associated with a high rate of cardiovascular mortality due to increased risks of hypertension, ischemic heart disease, heart failure and arrhythmia (1-3). Chronic kidney disease (CKD) is also established as a risk factor for cardiovascular morbidity and mortality $(4,5)$. OSAS and CKD might interact with and influence each other, as both are associated with common comorbidities such as hypertension, obesity and diabetes mellitus $(2,6,7)$. Indeed, several reports have demonstrated a relationship between OSAS and renal dysfunction. Patients with OSAS often have increased levels of urinary albumin excretion, glomerular hyperfiltration and proteinuria (8-10), and inversely, patients with end-stage renal disease often exhibit a high prevalence of OSAS (11).

Nasal continuous positive airway pressure (CPAP) is a popular medical device used to improve nocturnal desaturation and symptoms such as headaches, snoring and daytime sleepiness while decreasing blood pressure in patients with OSAS (12). Therapy with CPAP also protects against death from cardiovascular diseases and can improve mortality in patients with OSAS (1). However, whether CPAP adminis- 
Table 1. Clinical Characteristics

\begin{tabular}{|c|c|}
\hline & $\mathrm{n}=38$ \\
\hline Age (years) & $55 \pm 10$ \\
\hline Body mass index $\left(\mathrm{kg} / \mathrm{m}^{2}\right)$ & $28.1 \pm 4.4$ \\
\hline Hypertension & $22(58 \%)$ \\
\hline Anti-hypertensive medications & $20(53 \%)$ \\
\hline Calcium-channel blocker & $14(42 \%)$ \\
\hline ACE inhibitor & $6(16 \%)$ \\
\hline Angiotensin II-receptor blocker & $13(34 \%)$ \\
\hline$\beta$-blocker & 0 \\
\hline$\alpha$-blocker & $5(13 \%)$ \\
\hline Diuretics & 0 \\
\hline Systolic blood pressure (mmHg) & $126 \pm 16$ \\
\hline Diastolic blood pressure $(\mathrm{mmHg})$ & $78 \pm 10$ \\
\hline Heart rate (beats/min) & $70 \pm 11$ \\
\hline \multicolumn{2}{|l|}{ Lipid profiles (mg/dL) } \\
\hline Total cholesterol & $191 \pm 32$ \\
\hline LDL cholesterol & $113 \pm 25$ \\
\hline HDL cholesterol & $44 \pm 11$ \\
\hline Triglyceride & $178 \pm 148$ \\
\hline Diabetes mellitus & $10(26 \%)$ \\
\hline Current smoker & $8(21 \%)$ \\
\hline Serum creatinine $(\mathrm{mg} / \mathrm{dL})$ & $0.83 \pm 0.10$ \\
\hline $\mathrm{eGFR}\left(\mathrm{mL} / \mathrm{min} / 1.73 \mathrm{~m}^{2}\right)$ & $77.3 \pm 12.0$ \\
\hline \multicolumn{2}{|l|}{ Polysomnographic data } \\
\hline Apnea-hypopnea index (events/hour) & $39.0 \pm 22.1$ \\
\hline Lowest nocturnal oxygen saturation (\%) & $79.6 \pm 8.2$ \\
\hline Mean apnea duration (sec) & $23 \pm 10$ \\
\hline Hypoxemia index $(\%)$ & $7.9 \pm 12.7$ \\
\hline
\end{tabular}

tered to treat OSAS can affect CKD remains unclear.

This study was designed to clarify the association between OSAS and the estimated glomerular filtration rate (eGFR), a key indicator of CKD, and to investigate whether short-term CPAP therapy can improve the eGFR.

\section{Materials and Methods}

\section{Patients}

We enrolled 38 consecutive men with OSAS who were diagnosed based on a history of snoring, daytime sleepiness and apnea-hypopnea indices $\geq 5$ events/hour according to overnight polysomnography. The exclusion criteria comprised apparent lung diseases, daytime hypoxemia (arterial oxygen pressure $<80 \mathrm{mmHg}$ ), ischemic or valvular heart disease, changes in medications during the follow-up period and previous CPAP use. The Institutional Ethics Committee at our hospital approved the study, which conformed to the Declaration of Helsinki. Informed consent was obtained from each patient prior to their enrollment in the study.

\section{Sleep study and CPAP}

All patients underwent overnight polysomnography (EMBLA S7000; Medcare Flaga, Reykjavik, Iceland). Polysomnography consisted of 4-channel electroencephalography, electrooculography, submental and leg electromyography, electrocardiography and measurement of oronasal airflow (thermistors), chest and abdominal respiratory movements, snoring (microphone) and body position. Oxygen saturation was measured with a pulse oximeter. Apnea was defined as air flow cessation for $>10$ seconds. Hypopnea was defined as a $>50 \%$ reduction in respiratory signals for at least 10 seconds with 3\% desaturation from baseline. The total number of incidents of obstructive apnea/hypopnea had to exceed five per hour (apnea-hypopnea index, AHI) and the presence of clinical symptoms of OSAS was required for a diagnosis of OSAS. The lowest nocturnal oxygen saturation and the mean apnea duration were measured. The hypoxemia index was defined as the percentage of sleep time at oxygen saturation $<90 \%$. Patients with OSAS whose AHI was $\geq 20$ events/hour were prescribed CPAP (PV10; Breas, Molnlycke, Sweden) in fixed-pressure mode. Patients with an average CPAP use $<3.5$ hours/night were considered to be non-compliant and thus were excluded. All patients underwent clinical reassessment and a biochemical analysis after three months of CPAP.

\section{Measurements}

We calculated the eGFR based on age and the serum creatinine level using the Japanese eGFR estimation equation proposed by the Japanese Society of Nephrology (13). Hypertension was defined as a systolic blood pressure $\geq 140$ $\mathrm{mmHg}$ or a diastolic blood pressure $\geq 90 \mathrm{mmHg}$ and/or the use of anti-hypertensive medications. Body mass index (BMI) was calculated as weight/height ${ }^{2}\left(\mathrm{~kg} / \mathrm{m}^{2}\right)$. Patients with fasting plasma glucose levels exceeding $126 \mathrm{mg} / \mathrm{dL}$ and/or those under medication for diabetes or who were diagnosed with diabetes were defined as having diabetes mellitus.

\section{Statistical analysis}

All continuous values are expressed as the mean \pm SD, and their differences were assessed using paired t-tests. Categorical variables are reported as numbers and percentages. Univariate and multivariate linear regression analyses were used to determine the independent factors associated with eGFR. According to the univariate analysis, age, BMI, hypertension, diabetes mellitus, AHI, lowest nocturnal oxygen saturation, mean apnea duration and hypoxemia index were evaluated as explanatory variables. Specified variables with $\mathrm{p}<0.05$ in the univariate model were entered into the multivariate model. A $p$ value $<0.05$ was considered statistically significant. The data were analyzed using the SPSS 11.0 statistical software program (SPSS, Inc., Chicago, IL, USA).

\section{Results}

Table 1 lists the clinical characteristics, eGFR values and baseline polysomnographic data for the OSAS patients. The mean serum creatinine level was $0.83 \pm 0.10 \mathrm{mg} / \mathrm{dL}$ and the mean eGFR level was $77.3 \pm 12.0 \mathrm{~mL} / \mathrm{min} / 1.73 \mathrm{~m}^{2}$. 
Table 2. Univariate and Multivariate Analysis for eGFR Level

\begin{tabular}{lccccccc}
\hline & \multicolumn{3}{c}{ Univariate } & & \multicolumn{3}{c}{ Multivariate } \\
\cline { 2 - 4 } \cline { 7 - 8 } & $\beta$ & $95 \% \mathrm{CI}$ & $\mathrm{p}$ value & & $\beta$ & $95 \% \mathrm{CI}$ & $\mathrm{p}$ value \\
\hline Age & -0.69 & $-1.14--0.54$ & $<0.001$ & & -0.61 & $-1.05--0.45$ & $<0.001$ \\
Mean apnea duration & -0.44 & $-0.93--0.17$ & 0.006 & & -0.25 & $-0.63--0.003$ & 0.048 \\
Body mass index & 0.37 & $0.16-1.88$ & 0.022 & & & \\
\hline eGFR: estimated glomerular filtration rate, CI: confidence interval
\end{tabular}

Table 3. Changes in OSAS after 3 Months of CPAP

\begin{tabular}{lccc}
\hline & Baseline & After CPAP & p value \\
\hline Apnea-hypopnea index (events/hour) & $41.8 \pm 19.8$ & $5.7 \pm 6.3$ & $<0.001$ \\
Lowest nocturnal oxygen saturation (\%) & $77.9 \pm 7.7$ & $89.4 \pm 5.7$ & $<0.001$ \\
Hypoxemia index (\%) & $10.2 \pm 14.6$ & $0.48 \pm 1.2$ & 0.004 \\
Mean apnea duration (sec) & $25 \pm 12$ & $15 \pm 12$ & 0.011 \\
& & & \\
Body mass index (kg/m ${ }^{2}$ ) & $28.6 \pm 4.5$ & $27.9 \pm 4.2$ & 0.11 \\
Systolic blood pressure (mmHg) & $127 \pm 15$ & $128 \pm 17$ & 0.56 \\
Diastolic blood pressure (mmHg) & $79 \pm 11$ & $78 \pm 8$ & 0.60 \\
Heart rate (beats/min) & $69 \pm 11$ & $67 \pm 10$ & 0.26 \\
Lipid profiles (mg/dL) & & & \\
$\quad$ Total cholesterol & $189 \pm 31$ & $185 \pm 31$ & 0.46 \\
$\quad$ LDL cholesterol & $115 \pm 27$ & $108 \pm 31$ & 0.16 \\
$\quad$ HDL cholesterol & $45 \pm 11$ & $46 \pm 11$ & 0.80 \\
$\quad$ Triglyceride & $161 \pm 106$ & $170 \pm 93$ & 0.66 \\
Left ventricular ejection fraction & $0.67 \pm 0.04$ & $0.68 \pm 0.04$ & 0.87 \\
\hline OSAS: obstructive sleep apnea syndrome, CPAP: nasal continuous positive airway \\
pressure, HDL: high-density lipoprotein, LDL: low-density lipoprotein &
\end{tabular}

Using a linear regression analysis, we evaluated the significant factors associated with eGFR (Table 2). The univariate analysis revealed that an older age, a longer mean apnea duration and BMI were significantly associated with lower eGFRs. The multivariate analysis showed that the independent factors associated with lower eGFRs were older age and a longer mean apnea duration (Table 2).

Thirty-two of the original 38 OSAS patients had an AHI $\geq 20$ events/hour and were prescribed CPAP. Five of those patients were not compliant with CPAP treatment and withdrew from the study. The remaining 27 compliant patients were reassessed after three months of CPAP treatment. All polysomnographic data improved (Table 3). None of the values of BMI, blood pressure, heart rate, lipid profiles or ejection fraction changed after CPAP treatment. Figure shows the changes in the levels of serum creatinine and eGFR before and after three months of CPAP treatment. There were significant decreases in the serum creatinine levels $(0.87 \pm$ 0.09 to $0.82 \pm 0.01 \mathrm{mg} / \mathrm{dL}, \mathrm{p}=0.013$ ) and increases in eGFR ( $72.9 \pm 12.0$ to $\left.79.3 \pm 17.9 \mathrm{~mL} / \mathrm{min} / 1.73 \mathrm{~m}^{2}, \mathrm{p}=0.014\right)$ (Figure).

\section{Discussion}

The major finding of our study is that OSAS, especially that associated with an older age and a longer mean apnea duration, is significantly associated with decreased eGFRs, which can be reversed by CPAP treatment.

The following pathogenic mechanisms make it conceivable that OSAS may contribute to decreases in eGFR. First, nocturnal hypoxia is an underlying condition in patients with OSAS. Chronic hypoxia can lead to tubulointerstitial damage, tubular atrophy, development of atubular glomeruli and tubulointerstitial fibrosis (14). Second, sympathetic activity is high in patients with OSAS during wakefulness and further increases during sleep (15). High levels of renal sympathetic nerve activation, evoked reflexively by severe hypoxia, result in preglomerular vasoconstriction and a reduced glomerular ultrafiltration coefficient, with consequent reductions in eGFR (16). Third, OSAS patients also experience systemic inflammation, pressure surges and oxidative stress, all of which might contribute to the development of endothelial dysfunction (17). Endothelial dysfunction in glomeruli might lead to a decreased eGFR. Therefore, it is reasonable to speculate that OSAS contributes to decreases in eGFR.

Hypertension, obesity and diabetes mellitus are important risk factors for CKD (18-20). In addition, these factors are often seen in patients with OSAS. Laboratory and epidemiologic studies have shown that insufficient sleep and poor sleep quality promote the development and exacerbation of these risk factors (21). Therefore, attention should be paid to the influence of these confounding factors when discussing the relationship between OSAS and lower values of eGFR. In the present study, the multivariate regression analysis showed that hypertension is not an independent factor associated with eGFR. These findings might be influenced by the high proportion of patients taking anti-hypertensive medications with well-controlled blood pressure. Therefore, our findings cannot exclude the possibility that hypertension affected the eGFR values observed in this study. Additionally, pathological evidence obtained from kidney biopsy specimens supports the emergence of obesity-related 
A

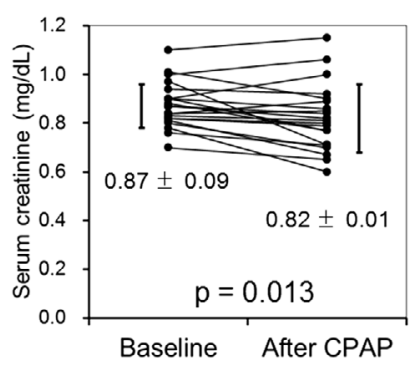

B

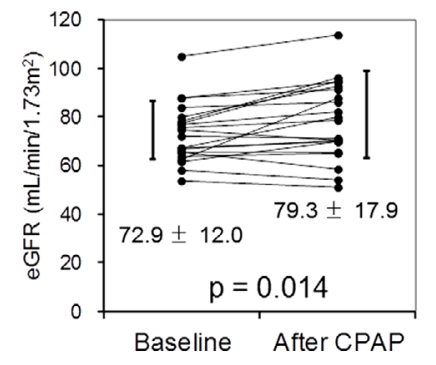

Figure. Changes in the serum creatinine levels (A) and estimated glomerular filtration rates (eGFRs) (B) at baseline and three months after nasal continuous positive airway pressure (CPAP) treatment.

glomerulopathy (22). However, the multivariate analysis in this study did not show any significant relationships between BMI and eGFR. To clarify this issue, further studies including BMI-matched control subjects are needed.

There are conflicting results regarding the eGFR values in patients with OSAS. Sakaguchi et al. (23) reported a high prevalence $(65 \%)$ of OSAS among non-dialysis CKD patients (median eGFR value: $28.5 \mathrm{~mL} / \mathrm{min} / 1.73 \mathrm{~m}^{2}$ ) in Japan. These authors also demonstrated that decreases in eGFR are significantly correlated with increases in AHI and are significant predictors of OSAS (23). In the present study, eGFR was found to be inversely correlated with the mean apnea duration, but not with AHI. On the other hand, Iseki et al. (24) detected CKD in $30.5 \%$ of patients with sleepdisordered breathing. Kinebuchi et al. (10) found that OSAS is not associated with decreased eGFR, but rather with elevated filtration fractions, thus implying the presence of glomerular filtration overload. The dissimilarities between the present and published findings might be due to heterogeneity in the inclusion criteria, sample sizes and severities of OSAS, obesity and hypertension.

In the present study, three months of CPAP therapy ameliorated decreased eGFR values. Although the degrees of improvement observed in the levels of serum creatinine and eGFR were subtle, the differences were statistically significant. There are some possible explanations for the CPAPinduced amelioration of decreased eGFR. Treatment with CPAP might protect glomeruli and reverse decreased eGFR by abolishing apneic and/or hypopneic episodes, maintaining normal oxygenation, correcting sympathetic activity and reversing endothelial dysfunction $(15,25)$. In the present study, we did not directly evaluate changes in sympathetic activity or endothelial dysfunction. However, all polysomnographic parameters significantly improved following CPAP therapy. BMI, blood pressure and initial antihypertensive medications did not change before and after CPAP. Taken together, it is reasonable to speculate that improvements in eGFR might be induced by amelioration of OSAS itself by CPAP. Our data are somewhat unique in that the eGFR values improved over a short period of three

months. This might be explained by the fact that the eGFR values were not very low and the severity of OSAS, obesity and hypertension was not very high in our patients.

There are several limitations associated with our study. First, this study is small and non-randomized. Second, we should have included control subjects who had undergone polysomnography and were proven to not have OSAS and matched for age, blood pressure, BMI, the serum creatinine levels and eGFR. Third, several factors, including inflammation, oxidative stress and/or endothelial dysfunction, are thought to promote decreases in eGFR by inducing OSAS, as mentioned above; however, we did not identify these contributing factors in this study. Identifying such factors would require obtaining data for serum high-sensitivity $\mathrm{C}$-reactive protein as an inflammatory marker, urinary 8-isoprostane and serum asymmetric dimethylarginen as oxidative stress markers, urinary L-type fatty acid binding protein, urinary $\mathrm{N}$-acetyl- $\beta$-D-glucosaminidase and serum beta- 2 microglobulin as markers of tubulointerstitial damage and measurements of flow-mediated dilation of the brachial artery in order to evaluate endothelial dysfunction. Additionally, we did not measure the serum or urinary levels of catecholamines or blood pressure during sleep; therefore, the influence of nocturnal changes on sympathetic nervous system activity remains unknown. Fourth, because all of the patients were men, the results cannot be applied to women. Fifth, since we did not perform urinalyses or measure the urinary albumin levels, the possibility that some patients with normal eGFR levels had subclinical renal damage cannot be excluded. Finally, since the eGFR values in our limited cohort were not very low, the results cannot be applied to advanced and end-stage CKD patients. Further studies including more patients with higher serum creatinine levels and/or CKD patients are therefore needed.

In conclusion, OSAS, especially that associated with an older age and a longer mean apnea duration, may contribute to lowering eGFR values, which can be reversed by CPAP treatment. To clarify this relationship, further randomized multicenter prospective studies with a larger number of subjects including controls matched for confounding factors are warranted.

The authors state that they have no Conflict of Interest (COI).

\section{References}

1. Marin JM, Carrizo SJ, Vicente E, Agusti AG. Long-term cardiovascular outcomes in men with obstructive sleep apnoeahypopnoea with or without treatment with continuous positive airway pressure: an observational study. Lancet 365: 1046-1053, 2005.

2. Peppard PE, Young T, Palta M, Skatrud J. Prospective study of the association between sleep-disordered breathing and hypertension. N Engl J Med 342: 1378-1384, 2000.

3. Shepard JW Jr. Hypertension, cardiac arrhythmias, myocardial infarction, and stroke in relation to obstructive sleep apnea. Clin Chest Med 13: 437-458, 1992. 
4. National Kidney Foundation. K/DOQI clinical practice guidelines for chronic kidney disease: evaluation, classification, and stratification. Am J Kidney Dis 39: S1-S266, 2002.

5. Go AS, Chertow GM, Fan D, McCulloch CE, Hsu CY. Chronic kidney disease and the risks of death, cardiovascular events, and hospitalization. N Engl J Med 351: 1296-1305, 2004.

6. Elmasry A, Lindberg E, Berne C, et al. Sleep-disordered breathing and glucose metabolism in hypertensive men: a population-based study. J Intern Med 249: 153-161, 2001.

7. Kyzer S, Charuzi I. Obstructive sleep apnea in the obese. World J Surg 22: 998-1001, 1998.

8. Casserly LF, Chow N, Ali S, Gottlieb DJ, Epstein LJ, Kaufman JS. Proteinuria in obstructive sleep apnea. Kidney Int 60: 14841489, 2001.

9. Faulx MD, Storfer-Isser A, Kirchner HL, Jenny NS, Tracy RP, Redline S. Obstructive sleep apnea is associated with increased urinary albumin excretion. Sleep 30: 923-929, 2007.

10. Kinebuchi S, Kazama JJ, Satoh M, et al. Short-term use of continuous positive airway pressure ameliorates glomerular hyperfiltration in patients with obstructive sleep apnoea syndrome. Clin Sci (Lond) 107: 317-322, 2004.

11. Kimmel PL, Miller G, Mendelson WB. Sleep apnea syndrome in chronic renal disease. Am J Med 86: 308-314, 1989.

12. Davies RJ, Crosby J, Prothero A, Stradling JR. Ambulatory blood pressure and left ventricular hypertrophy in subjects with untreated obstructive sleep apnoea and snoring, compared with matched control subjects, and their response to treatment. Clin Sci (Lond) 86: 417-424, 1994.

13. Iseki K. Chronic kidney disease in Japan from early predictions to current facts. Nephron Clin Pract 110: c268-c272, 2008.

14. Nangaku M. Chronic hypoxia and tubulointerstitial injury: a final common pathway to end-stage renal failure. J Am Soc Nephrol 17: 17-25, 2006.

15. Somers VK, Dyken ME, Clary MP, Abboud FM. Sympathetic neural mechanisms in obstructive sleep apnea. J Clin Invest 96:
1897-1904, 1995.

16. Denton KM, Shweta A, Anderson WP. Preglomerular and postglomerular resistance responses to different levels of sympathetic activation by hypoxia. J Am Soc Nephrol 13: 27-34, 2002.

17. Kato M, Roberts-Thomson P, Phillips BG, et al. Impairment of endothelium-dependent vasodilation of resistance vessels in patients with obstructive sleep apnea. Circulation 102: 2607-2610, 2000.

18. Fox CS, Larson MG, Leip EP, Culleton B, Wilson PW, Levy D. Predictors of new-onset kidney disease in a community-based population. JAMA 291: 844-850, 2004.

19. Gelber RP, Kurth T, Kausz AT, et al. Association between body mass index and CKD in apparently healthy men. Am J Kidney Dis 46: 871-880, 2005.

20. Young JH, Klag MJ, Muntner P, Whyte JL, Pahor M, Coresh J. Blood pressure and decline in kidney function: findings from the Systolic Hypertension in the Elderly Program (SHEP). J Am Soc Nephrol 13: 2776-2782, 2002.

21. Turek NF, Ricardo AC, Lash JP. Sleep disturbances as nontraditional risk factors for development and progression of CKD: review of the evidence. Am J Kidney Dis 2012 (in press).

22. Kambham N, Markowitz GS, Valeri AM, Lin J, D'Agati VD. Obesity-related glomerulopathy: an emerging epidemic. Kidney Int 59: 1498-1509, 2001.

23. Sakaguchi Y, Shoji T, Kawabata H, et al. High prevalence of obstructive sleep apnea and its association with renal function among nondialysis chronic kidney disease patients in Japan: a crosssectional study. Clin J Am Soc Nephrol 6: 995-1000, 2011.

24. Iseki K, Tohyama K, Matsumoto T, Nakamura H. High prevalence of chronic kidney disease among patients with sleep related breathing disorder (SRBD). Hypertens Res 31: 249-255, 2008.

25. Schulz R, Schmidt D, Blum A, et al. Decreased plasma levels of nitric oxide derivatives in obstructive sleep apnoea: response to CPAP therapy. Thorax 55: 1046-1051, 2000.

(C) 2013 The Japanese Society of Internal Medicine http://www.naika.or.jp/imonline/index.html 